\section{RESEARCH HIGHLIGHTS: Why are COVID-19 vaccines still in short supply?}

\section{By Rachana Pradhan and Arthur Allen and Kaiser Health News}

$\mathrm{T}^{\mathrm{s}}$ he US Government has invested billions of dollars in manufacturing, used the Defense Production Act (DPA) dozens of times to boost supplies and yet there is still not enough COVID-19 vaccine on the way to meet demand-or even the government's own goals for national immunization.

Experts say it is complicated, noting that the output of raw materials and assembly lines cannot be ratcheted up 10,000 -fold at the push of a buttonand that the effort thus far has been close to miraculous. They cite bottlenecks in at least three areas: the production of specialty lipids, fatty materials that are a primary component of the Moderna and Pfizer-BioNTech vaccines; the hundreds of millions of glass vials that hold the vaccine; and the sterile automated assembly lines where vaccine moves from bulk containers into vials before shipment.

Also, experts note, no one knew which vaccines would prove effective.

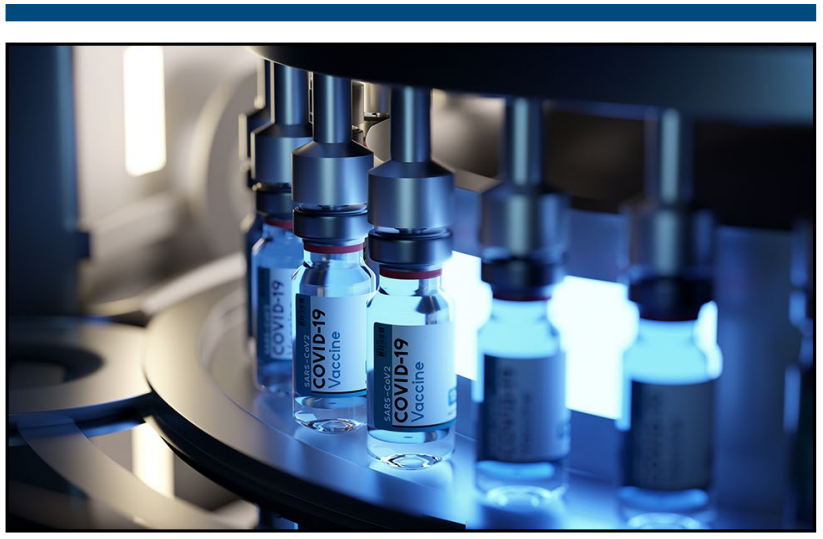

"A year ago there was no commercial market for mRNA product. There was scientific research and pharma making small-volume clinical lots. Now we need billions of doses, in the space of a year. That's overloading the supply infrastructure," said Kevin Gilligan, a senior consultant with Biologics Consulting and a former official with the Biomedical Advanced Research and Development Authority, or BARDA, a US federal agency created in 2006 to deal with pandemics and bioterrorism.

The DPA has been used in vaccine contracts with Moderna, Inc. (cofounded by materials researcher Robert Langer of the Massachusetts Institute of Technology), Johnson \& Johnson, and other drug companies for hundreds of millions of doses. On top of that, the law has been invoked for at least 10 contracts with companies making needles or syringes. It has been used to require glassmakers Corning Incorporated and $\mathrm{SiO} 2$ Materials Science to prioritize vial production for vaccine production, and in contracts for aspects of manufacturing.

Each PfizerBioNTech or Moderna shot contains billions of lipid nanoparticles, each particle containing four lipids and a strand of the nucleic acid RNA, with the five pieces assembled in a way that allows the RNA to enter our cells and create a particle that stimulates the immune system to defend against the COVID-19 virus.

The lipids, which are made only in a handful of factories, have been a major supply problem. "No one has ever thought of a scenario where we would use lipid nanoparticle formulation for [billions of] doses," says Prashant Yadav, a senior fellow at the Center for Global Development at Harvard University. "We have not invented a process for doing lipid nanoparticles at scale."

Two of the lipids in the vaccine, cholesterol and DSCP (a phospholipid), have long been used in industry to shape and buffer chemical formulations. A third lipid prevents the particles from clumping together. A fourth enables the lipid shell of the vaccine to fuse with human cells and, once inside the cell, to crack open so the RNA can move to the ribosome and make proteins that stimulate immunity.

All of these raw materials are produced under regulated conditions - in Massachusetts, Missouri, Colorado, and Alabama by companies under license with Moderna, Inc., Pfizer, Inc., or Acuitas Therapeutics, which was cofounded by Pieter Cullis, a University of British Columbia (UBC) professor who is considered the grandfather of lipid nanoparticle technology.

Before the pandemic, these companies produced meager amounts for use in small clinical trials, laboratory experiments, or in one licensed drug, patisiran, which is used to treat a rare genetic disease in about a thousand people worldwide. Now they are producing thousands of kilograms of the stuff, says Stefan Randl, a vice president at Evonik, a lipid maker. Evonik recently announced it would scale up production at two German sites, possibly in

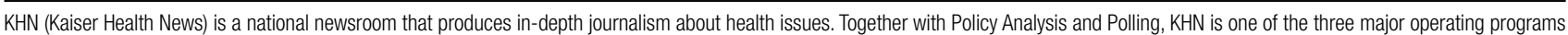
at KFF (Kaiser Family Foundation). KFF is an endowed nonprofit organization providing information on health issues to the nation. 
the second half of the year, to be used in the Pfizer-BioNTech vaccine. The company last year bought a US lipid manufacturer in Alabama.

"All of a sudden the quantities had to be ramped up a thousandfold or more," Randl says. "This is the biggest bottleneck."

Several elements of the vaccine, including lipids and enzymes used in making the mRNA, until recently were produced using animal products such as sheep's wool, says Andrew Geall, chief scientific officer at Precision NanoSystems Inc., which designs equipment for mixing the mRNA and lipids. Animal products could cause contamination or disease, even in minute quantities, so manufacturers now use synthetic chemicals.

Luckily, the cosmetic industry-a major user of some of the same lipids used in the vaccines- has been switching from animal products in recent decades, notes Julia Born, an Evonik spokesperson.

Still, only a limited number of companies globally have expertise and facilities to make the lipids, says Thomas Madden, CEO and a co-founder of
Acuitas Therapeutics, and they have all struggled to move from quantities produced in a laboratory to industrialscale production. For instance, he says, hazardous solvents and chemicals used in laboratory procedures need to be avoided in industrial processes, where they could give rise to workplace safety issues.

"This is a hugely complex supply chain," Madden says. "Once you address a bottleneck at one point, you identify the next bottleneck in the process. It's a bit of a game of whack-a-mole."

Although it is not particularly difficult to make the lipids used in vaccines, it takes time to get US Food and Drug Administration (FDA) authorization for a facility that can make them in high quantities, says Cullis, the UBC professor. It would take two to three years to start such a factory from scratch, so instead, Moderna and Pfizer-BioNTech have been working with existing manufacturers and getting them to convert to lipid production, he says.

Another bottleneck is "fill/finish"getting the finished vaccine into vials or syringes so the shots can be shipped to customers. Vaccine filling lines require extremely high levels of efficiency and sterility, and few companies in the world have this capacity, says Mike Watson, former president of Valera, a Moderna subsidiary. Moderna has hired Catalent, Inc., a contract manufacturer that recently experienced delays that slowed the release of some doses, to fill and finish US doses at its facility in Bloomington, Ind. At least two other companies will do the same for Moderna's vaccine supply abroad.

In January, the French multinational Sanofi-whose own COVID19 vaccine has been delayed by poor performance in producing immunity - agreed to offer its fill/finish line in Germany for the Pfizer-BioNTech vaccine. That line is not expected to be running until July.

In the United States, the number of vaccine doses shipped to states has ticked up in recent weeks, partly because Pfizer said its five-dose vials actually provide six shots. At the time of this report, Moderna was seeking FDA permission to add up to five doses to its 10-dose vials.

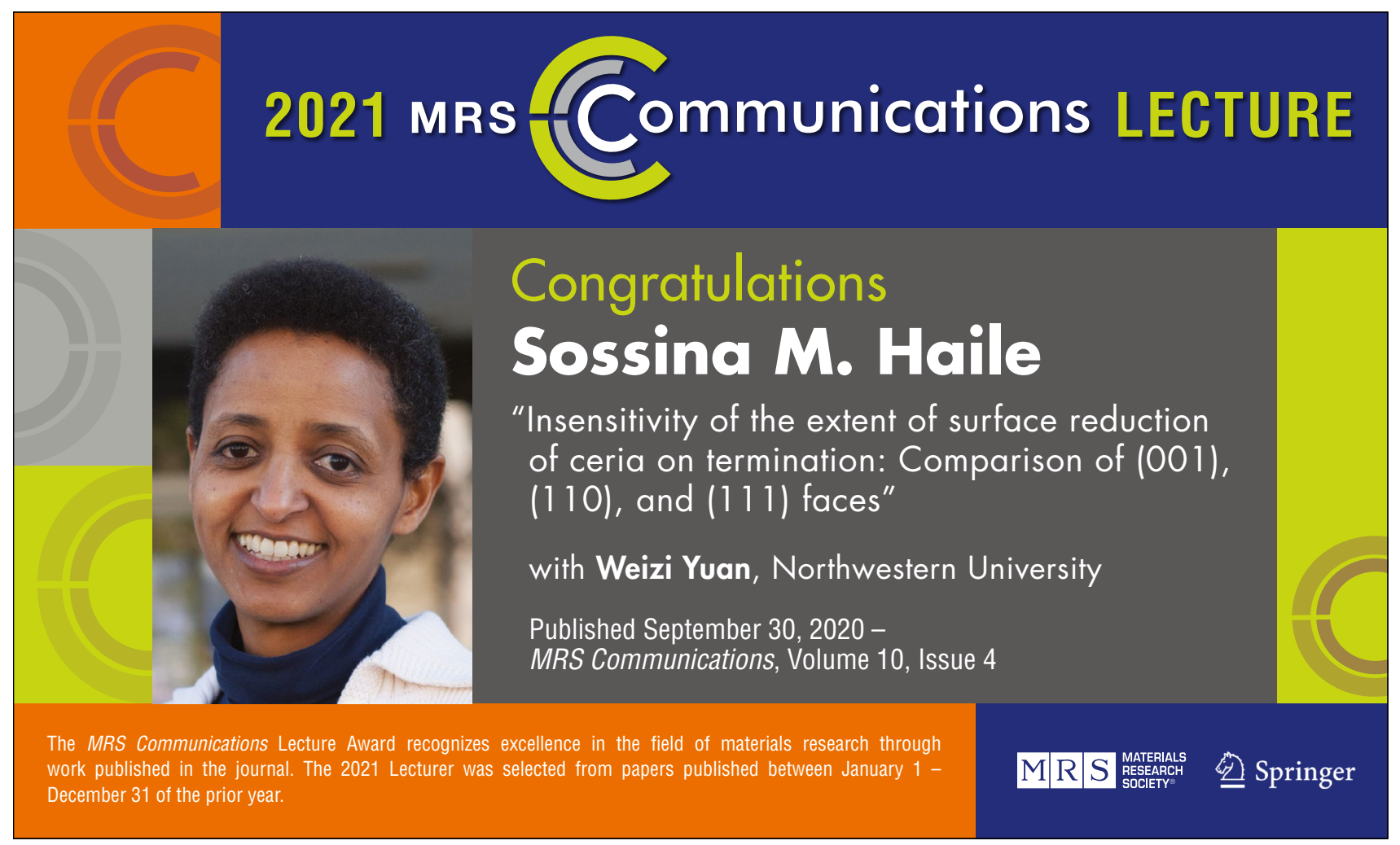

